\title{
Verursacht langjährige Asbestexposition eine obstruktive Ventilationsstörung?
}

\author{
Does Long-Term Asbestos Exposure Cause an Obstructive Ventilation Pattern?
}

Autoren

Institut
X. Baur, U. Manuwald, D. Wilken

Ordinariat für Arbeitsmedizin, Universitätsklinikum Hamburg-Eppendorf, Zentralinstitut für Arbeitsmedizin und Maritime Medizin eingereicht 5. 3. 2010

akzeptiert nach Revision 1. 6.2010

\section{Bibliografie}

DOI http://dx.doi.org/ 10.1055/s-0030-1255555

Online-Publikation: 15. 7. 2010

Pneumologie 2010; 64:

736-744 @ Georg Thieme

Verlag KG Stuttgart · New York ISSN 0934-8387

Korrespondenzadresse Prof. Dr. med. X. Baur Ordinariat für Arbeitsmedizin Universitätsklinikum HamburgEppendorf

Zentralinstitut für Arbeitsmedizin und Maritime Medizin Seewartenstr. 10

20459 Hamburg baur@uke.de

\section{Zusammenfassung \\ $\nabla$}

Die Literaturübersicht zeigt, dass langjährige Exposition gegenüber asbestfaserhaltigen Stäuben neben der Verminderung der Lungenvolumina zu einer Einschränkung der forcierten Atemflüsse, v.a. der $\mathrm{FEF}_{50}$ und $\mathrm{FEF}_{75}$, aber auch überhäufig der $\mathrm{FEV}_{1} / \mathrm{FVC}$ sowie einer Erhöhung des Atemwegswiderstands führt. Es ergeben sich signifikante Dosis-Wirkungsbeziehungen und eine Zunahme der Funktionsstörungen bei langer Latenzzeit. Bemerkenswerterweise weisen bereits Kollektive ohne radiologisch fassbare Pleura- und Lungenparenchymveränderungen weitestgehend konsistent diese Veränderungen auf. Unter Nichtrauchern sind die asbestbedingten Lungenfunktionseinschränkungen im Mittel gering, im Einzelfall können sie aber den pathologischen Bereich und klinische Relevanz erreichen. Unter rauchenden Asbestexponierten sind die Lungenfunktionseinschränkungen in Folge synergistischer Wirkungen vor allem im Bereich der peripheren Atemwege hoch signifikant ausgeprägt. Die Verwendung nicht zeitgemäßer Sollwerte, Healthy-worker-Effekte und die Lungenblähung unterschätzen die asbestbedingten Lungenfunktionsveränderungen. Zwischen den verschiedenen untersuchten Berufsgruppen mit Asbestexposition ist kein eindeutiger Unterschied festzustellen.

\section{Einleitung}

Neuere Untersuchungen belegen, dass die Arbeits- und allgemeine Umweltexposition gegenüber Stäuben mit ihren variablen Anteilen an einatembarer und alveolengängiger Fraktion dosisabhängig mit einem erhöhten Risiko für eine COPD und Lungenkrebs assoziiert ist [1 -6]. Hinzu kommen spezifische Effekte einzelner Noxen, z. B. Zellläsionen durch Asbestfasern im Rahmen deren lebenslanger Wanderung durch das Lungengewe-

\section{Abstract \\ $\nabla$}

This review shows that long-term exposure to asbestos-containing dust leads not only to a reduction of lung volume as well as to limitations of forced expiratory flows, such as $\mathrm{FEF}_{50}$ and $\mathrm{FEF}_{75}$, but also to increased frequencies of $\mathrm{FEV}_{1} /$ FVC, and elevated airway resistance. There is evidence for significant dose-response relationships and an increase in functional changes in parallel to an increase due to the latency period. Remarkably, even asbestos workers without radiologically detectable pleural or parenchymal changes already show these functional impairments. In nonsmokers, asbestos-induced lung function impairment is usually small on average, although some of these subjects show functional impairment of clinical relevance in the pathological range. In asbestos workers who also smoke, due to synergistic effects, lung function, especially of the peripheral airways, is highly significantly reduced. The use of inappropriate reference values, healthy worker effects, and airway trapping lead to an underestimation of asbestos-induced lung function impairments. There are no differences among the various occupations associated with asbestos exposure.

be, kanzerogene Wirkungen von Tabakrauchbestandteilen, polyzyklischen aromatischen Kohlenwasserstoffen, sechswertigem Chrom, Nickel, Asbest, Quarz etc. In welchem Umfang und unter welchen speziellen Gegebenheiten die Asbestexposition mit einer obstruktiven Ventilationsstörung einhergeht, wird kontrovers diskutiert [716]. Ziel dieser Arbeit ist eine Literaturaus- und -bewertung zu der Frage, ob in asbestexponierten Kollektiven eine obstruktive Lungenfunktionseinschränkung in Abhängigkeit vom vorliegenden 
radiologischen Befund, der beruflichen Tätigkeit und der Asbestfaserexposition (Dauer oder Dosis) festzustellen ist.

\section{Methode}

In Anlehnung an eine frühere Arbeit [17] erfolgte eine Aktualisierung und Spezifizierung der dort zugrunde gelegten PubMedLiteraturrecherche [18]. Mit Hilfe eines angepassten Suchlaufes konnten 191 relevante Arbeiten identifiziert werden. Nach einem Screening auf definierte Ausschlusskriterien entfielen 130 Arbeiten, da diese über keine Asbestexposition berichteten, keine Messwerte der Lungenfunktion enthielten oder nicht als Originalarbeit zugänglich waren. Die verbliebenen Arbeiten wurden anschließend auf die Erfüllung der Einschlusskriterien überprüft: Es musste mindestens ein Obstruktionsparameter $\left(\mathrm{FEV}_{1} / \mathrm{FVC}\right.$ oder ein Fluss-Volumen-Wert) in Bezug auf einen Sollwert angegeben worden sein; eine radiologische Untersuchung zur Ermittlung von asbestbedingten Lungenveränderungen war ebenfalls Voraussetzung; die Lungenfunktionswerte mussten den auf der Basis der radiologischen Untersuchung gebildeten Sub-Kollektiven zuzuordnen sein. Diese Anforderungen erfüllten 20 Arbeiten, die in die nachfolgende integrative Auswertung eingingen.

\section{Ergebnisse \\ $\nabla$}

1. Metaanalyse von Filippelli et al. [19]

Filippelli et al. [19] ( $\bullet$ Tab. 1 a-c) werteten in ihrer Metaanalyse 21 kontrollierte Studien mit insgesamt 24832 asbestexponierten Personen aus. Im Vergleich zu Kontrollpersonen waren neben restriktiven Veränderungen im Gesamtkollektiv signifikante Reduktionen von $\mathrm{FEV}_{1} / \mathrm{FVC}, \mathrm{PEF}, \mathrm{FEF}_{50}, \mathrm{FEF}_{25-75}$, TGV und RV/TGV sowie ein signifikanter Anstieg der Resistance (R) festzustellen ( $\odot$ Tab. 1 a). $\mathrm{FEV}_{1} / \mathrm{FVC}, \mathrm{FEF}_{25-75}, \mathrm{TGV}, \mathrm{RV} / \mathrm{TGV}$ und Resistance zeigten gleichartige signifikante Abweichungen auch unter nichtrauchenden Asbestarbeitern ( $\bullet$ Tab. 1 b). $\mathrm{FEF}_{25-75}$, TGV und RV/ TGV waren signifikant stärker unter rauchenden als unter nichtrauchenden Exponierten verändert ( $\bullet$ Tab. 1c).

Im Folgenden werden ergänzend Untersuchungen mit Angabe numerischer Lungenfunktionswerte und Berücksichtigung des radiologischen Befundes dargestellt, die in die vorgenannte Metaanalyse nicht eingingen.

\section{2. $F E V_{1} / F V C$ einzelner Studien}

In einer frühen Übersichtsarbeit [20] wurden bereits neben restriktiven Funktionseinschränkungen Befunde einer Obstruktion in asbestexponierten Kollektiven beschrieben (in den weitgehend linearen Dosis-Wirkungsbeziehungen der Nichtraucher und Raucher war die $\mathrm{FEV}_{1}$ stärker eingeschränkt als die FVC).

Die in $\triangle$ Abb. 1 wiedergegebenen neueren Literaturmitteilungen weisen konsistent und weitgehend unabhängig vom radiologischen Befund eine leichte, im Mittel vier- bis zwölf-prozentige Verminderung der $\mathrm{FEV}_{1} / \mathrm{FVC}$ aus.

Hier konnten mehrere asbestexponierte Kollektive wegen fehlender Bezüge auf Sollwerte nicht integriert werden. Dazu zählen Ohar et al. [8] (in 31,4\% $\mathrm{FEV}_{1} / \mathrm{FVC}<70 \%$ ), Rosenstock et al. [21] (in $21,7 \% \mathrm{FEV}_{1} / \mathrm{FVC}$ unter dem Grenzwert), Chien et al. [22] (in 35\% $\mathrm{FEV}_{1} / \mathrm{FVC}<70 \%$ ), Glencross et al. [23] (21\% obstruktive Ventilationsstörung) und Kennedy et al. [24], die in 21\% (unauffälliger Röntgenthorax), 33\% (Pleurafibrose) bzw. 19\% (Asbestose) eine Obstruktion und in jeweils in 8 bis $12 \%$ zusätzlich eine kombi- nierte Ventilationsstörung beobachteten. Für eine Obstruktion bei freiem kostophrenischen Winkel ergab sich eine OR von 2,59 (95\% CI 0,7-10), bei verschwartetem kostophrenischen Winkel betrug die OR 5,31 (95\% CI 1-28). Auch Schwartz et al. [25], Bourbeau et al. [26] und Ameille et al. [27] fanden eine ausgeprägtere Obstruktion, wenn der kostophrenische Winkel einbezogen war; letztere Autoren beschrieben in Kollektiven mit unterschiedlichen pleuralen Befunden eine Belastungsdyspnoe in $34,5-54,6 \%$ und eine chronische Bronchitis in 14,4-21,2\%. Nach Tonori et al. [28] nimmt die Häufigkeit obstruktiver Ventilationsstörungen bei zunehmendem Asbestosegrad ab (Grad $1=15,2 \%$, Grad $2=10 \%$, Grad $3=0 \%$ ).

Niebecker et al. [14] beschrieben ebenfalls signifikante Verminderungen der $\mathrm{FEV}_{1} / \mathrm{FVC}$ im Kollektiv mit Asbestose und/oder asbestbedingten Pleuraveränderungen. Dagegen war die $\mathrm{FEV}_{1} / \mathrm{FVC}$ Abnahme bei Begin et al. [29] mit ca. 90\% des Sollmittelwertes nicht signifikant.

Bagatin et al. [30] fanden eine signifikante Assoziation zwischen Asbestose und/oder asbestbedingten Pleuraveränderungen einerseits und obstruktiver Ventilationsstörung $\left(\mathrm{FEV}_{1} / \mathrm{FVC}<70 \%\right)$ andererseits (OR 2,2, $95 \%$ CI 1,55-3,11), 18-26\% waren obstruktiv.

Demers et al. [31] ermittelten nach mehr als 20-jähriger Asbestexposition eine Abnahme der $\mathrm{FEV}_{1} / \mathrm{FVC}$ auf $93,4 \%$ des Sollmittelwertes.

Des Weiteren lassen sich aus ergänzenden Angaben, insbesondere aus den z.T. nur numerisch ausgewiesenen, relativ stärkeren $\mathrm{FEV}_{1^{-}}$als FVC-Reduktionen auch ohne explizite Angaben der $\mathrm{FEV}_{1} /$ FVC-Sollwertabweichungen, in weiteren Veröffentlichungen leichte obstruktive Ventilationsstörungen in asbestexponierten Kollektiven ableiten [10,13,32-45]. Dement et al. [6] stellten unter asbestexponierten Bauarbeitern/Isolierern von US amerikanischen Nuklearwaffenbasen eine Prävalenz der COPD von 18,7\% (OR 2,66; 95\% CI 1,46-4,97) fest (Vergleichskollektiv 6,7\%); bei Nachweis von Pleuraveränderungen wurde in $23 \%$ und bei Nachweis von Pleura- und Parenchymveränderungen in 32,5\% in dem auch gegenüber anderen Stäuben belasteten Gesamtkollektiv eine COPD diagnostiziert. Ausnahmen hiervon stellen bei überwiegender Verwendung nicht zeitgemäßer Lungenfunktionssollwerte die Arbeiten von Begin et al. [46], Van Cleemput et al. [47], Schwartz et al. [25,48], Ameille et al. 2010 [49], Peric et al. [50] und Petrovic et al. [51] dar. Schwartz et al. [52] und Shih et al. [53] beschrieben lediglich bei Pleurafibrose (alle Grade zusammengefasst) bzw. bei diffuser Pleurafibrose eine reduzierte $\mathrm{FEV}_{1} / \mathrm{FVC}$.

Multiple lineare Regressionsanalysen zeigen, dass asbestbedingte Pleuraveränderungen mit einer Reduktion der $\mathrm{FEV}_{1} / \mathrm{FVC}$ assoziiert sind [24,54-57]; (Ausnahme: [47]). Entsprechendes gilt für das Vorliegen einer Asbestose [24,30,54-56,58]. In etwa der Hälfte dieser Untersuchungen liegen signifikante Ergebnisse auf Basis von (HR)CT-Befunden vor. Glencross et al. 1997 [23] beschrieben signifikante Assoziationen zwischen der asbestbelastenden Tätigkeit im Schiffsbau und dem $\mathrm{FEV}_{1}$-Abfall unter Rauchern sowie zwischen der Dauer der Asbestexposition und dem $\mathrm{FEV}_{1}$-Abfall unter Nichtrauchern.

\section{Flussvolumenwerte}

Auch die $\mathrm{FEF}_{25-75}$ zeigt weitgehend unabhängig von Röntgenbefunden im Mittel leichte Einschränkungen in asbestexponierten Kollektiven. Moshammer und Neuberger [59] beschrieben zusätzlich eine signifikante Korrelation zwischen Asbestfaserdosis und $\mathrm{FEF}_{75}$-Abnahme (Koeffizient: - 0,05; 95\% CI 0,10-0,005). 
Tab. 1 a Metaanalyse zur Lungenfunktion unter Asbestarbeitern, Einzelheiten s. Text (aus: Filippelli et al. 2008) [19]. Alle Exponierten (exp.) vs. nichtexponierte Kontrollen (Kontr.).

\begin{tabular}{|c|c|c|c|c|c|c|c|}
\hline \multirow[t]{2}{*}{ Parameter } & \multirow[t]{2}{*}{ Gruppe } & \multirow[t]{2}{*}{$\mathbf{n}$} & \multicolumn{5}{|c|}{ Metaanalyseindikatoren } \\
\hline & & & Ergebn. & $\mathrm{ES}, \mathrm{Z}$ & $\mathrm{I}^{2}, \%$ & REM/FEM & $\mathbf{P}$ \\
\hline \multirow[t]{2}{*}{ TGV } & exp. & 1476 & $\uparrow$ & 15,034 & - & WMD $0,833[0,725 ; 0,942]$ & $<0,001$ \\
\hline & Kontr. & 456 & & & & & \\
\hline \multirow[t]{2}{*}{ RV/TGV } & exp. & 738 & $\uparrow$ & 3,856 & 92,983 & SMD 1,190 [0,585; 1,795] & $<0,001$ \\
\hline & Kontr. & 228 & & & & & \\
\hline \multirow[t]{2}{*}{$\mathrm{FEV}_{1} / \mathrm{FVC}$} & exp. & 2615 & $\downarrow$ & 2,924 & 94,119 & SMD $-0,475[-0,794 ;-0,157]$ & $=0,003$ \\
\hline & Kontr. & 1561 & & & & & \\
\hline \multirow[t]{2}{*}{ PEF } & exp. & 233 & $\downarrow$ & 2,230 & 90,188 & SMD -0,979 [-1,838; - 0,119] & $=0,026$ \\
\hline & Kontr. & 215 & & & & & \\
\hline \multirow[t]{2}{*}{$\mathrm{FEF}_{50}$} & exp. & 400 & $\downarrow$ & 2,251 & 90,256 & SMD -0,651 [-1,219; -0,084] & $=0,024$ \\
\hline & Kontr. & 335 & & & & & \\
\hline \multirow[t]{2}{*}{$\mathrm{FEF}_{25-75}$} & exp. & 2838 & $\downarrow$ & 2,328 & 90,406 & SMD $-0,281[-0,517 ;-0,044]$ & $=0,020$ \\
\hline & Kontr. & 1242 & & & & & \\
\hline \multirow[t]{2}{*}{$\mathrm{R}$} & exp. & 33 & $\uparrow$ & 3,465 & 3,954 & WMD 0,910 [0,395; 1,435] & $=0,001$ \\
\hline & Kontr. & 39 & & & & & \\
\hline
\end{tabular}

$\uparrow$ : signifikant höher in der exponierten Gruppe; $\downarrow$ : signifikant erniedrigt in der exponierten Gruppe; WMD: gewichtete mittlere Differenz; SMD: standardisierte mittlere Differenz; $\left.\right|^{2}$ = Inkonsistenzmaß, ES = Effektgröße, REM = Random-Effekt-Modell, FEM = fixiertes Effektmodell.

Tab. 1b Metaanalyse zur Lungenfunktion unter Asbestarbeitern, Einzelheiten s. Text (aus: Filippelli et al. 2008) [19].

Exponierte Nichtraucher vs. nichtexponierte Nichtraucher.

\begin{tabular}{|c|c|c|c|c|c|c|}
\hline \multirow[t]{2}{*}{ Parameter } & \multirow[t]{2}{*}{ Gruppe } & \multirow[t]{2}{*}{$\mathbf{n}$} & \multirow[b]{2}{*}{ Ergebn. } & \multicolumn{2}{|c|}{ Metaanalyseindikatoren } & \multirow[b]{2}{*}{$\mathbf{P}$} \\
\hline & & & & $I^{2}, \%$ & REM/FEM & \\
\hline \multirow[t]{2}{*}{ TGV } & exp. & 310 & $\uparrow$ & - & WMD 0,701 [0,494; 0,909] & $<0,001$ \\
\hline & Kontr. & 134 & & & & \\
\hline \multirow[t]{2}{*}{ RV/TGV } & exp. & 155 & $\uparrow$ & - & WMD $0,843[0,546 ; 1,140$ & $<0,001$ \\
\hline & Kontr. & 67 & & & & \\
\hline \multirow[t]{2}{*}{$\mathrm{FEV}_{1} / \mathrm{FVC}$} & exp. & 264 & $\downarrow$ & 94,468 & SMD - $1,451[-2,406 ;-0,496]$ & $=0,003$ \\
\hline & Kontr. & 403 & & & & \\
\hline \multirow[t]{2}{*}{ PEF } & exp. & 18 & n.s. & - & WMD $-0,236[-0,433 ;-0,038]$ & $=0,562$ \\
\hline & Kontr. & 53 & & & & \\
\hline \multirow[t]{2}{*}{$\mathrm{FEF}_{50}$} & exp. & 49 & n.s. & 63,191 & SMD -0,334 [-0,946; 0,277] & $=0,284$ \\
\hline & Kontr. & 57 & & & & \\
\hline \multirow{2}{*}{$\mathrm{FEF}_{25-75}$} & exp. & 318 & $\downarrow$ & - & WMD $-0,158[-0,694 ;-0,377]$ & $=0,019$ \\
\hline & Kontr. & 155 & & & & \\
\hline \multirow[t]{2}{*}{$R$} & exp. & 27 & $\uparrow$ & - & WMD 1,089 [0,470; 1,707] & $=0,001$ \\
\hline & Kontr. & 20 & & & & \\
\hline
\end{tabular}

$\uparrow:$ signifikant höher in der exponierten Gruppe; $\downarrow$ : signifikant erniedrigt in der exponierten Gruppe; WMD: gewichtete mittlere Differenz; SMD: standardisierte mittlere Differenz; $I^{2}$ = Inkonsistenzmaß, REM = Random-Effekt-Modell, FEM = fixiertes Effektmodell.

Tab. 1c Metaanalyse zur Lungenfunktion unter Asbestarbeitern, Einzelheiten s. Text (aus: Filippelli et al. 2008) [19]. Exponierte Raucher (AR) vs. exponierte Nichtraucher (NR.).

\begin{tabular}{|c|c|c|c|c|c|c|}
\hline \multirow[t]{2}{*}{ Parameter } & \multirow[t]{2}{*}{ Gruppe } & \multirow[t]{2}{*}{$\mathbf{n}$} & \multicolumn{3}{|c|}{ Metaanalyseindikatoren } & \multirow[b]{2}{*}{$\mathbf{P}$} \\
\hline & & & Ergebn. & $I^{2}, \%$ & REM/FEM & \\
\hline TGV & $\begin{array}{l}\text { AR } \\
\text { NR. }\end{array}$ & $\begin{array}{r}1166 \\
620\end{array}$ & $\uparrow$ & 54,065 & SMD $0,267[0,169 ; 0,365]$ & $<0,001$ \\
\hline RV/TGV & $\begin{array}{l}\text { AR } \\
\text { NR. }\end{array}$ & $\begin{array}{l}583 \\
310\end{array}$ & $\uparrow$ & 93,599 & SMD $0,745[0,180 ; 1,309]$ & $=0,010$ \\
\hline $\mathrm{FEV}_{1} / \mathrm{FVC}$ & $\begin{array}{l}\text { AR } \\
\text { NR. }\end{array}$ & $\begin{array}{l}841 \\
361\end{array}$ & n.s. & 98,019 & SMD $1,118[-0,039 ; 2,276]$ & $=0,058$ \\
\hline PEF & $\begin{array}{l}\text { AR } \\
\text { NR. }\end{array}$ & $\begin{array}{l}124 \\
189\end{array}$ & n.s. & - & WMD $0,002[-0,492 ; 0,497]$ & $=0,992$ \\
\hline $\mathrm{FEF}_{50}$ & $\begin{array}{l}\text { AR } \\
\text { NR. }\end{array}$ & $\begin{array}{r}209 \\
26\end{array}$ & n.s. & 98,946 & SMD $-3,102[-9,212 ; 3,008]$ & $=0,320$ \\
\hline $\mathrm{FEF}_{25-75}$ & $\begin{array}{l}\text { AR } \\
\text { NR. }\end{array}$ & $\begin{array}{r}1251 \\
628\end{array}$ & $\downarrow$ & 94,541 & SMD $-0,281[-0,517 ;-0,044]$ & $=0,001$ \\
\hline $\mathrm{R}$ & $\begin{array}{l}\text { AR } \\
\text { NR. }\end{array}$ & $\begin{array}{l}19 \\
20\end{array}$ & n.s. & - & WMD 0,3154 [-0,574; 1,202] & $=0,488$ \\
\hline
\end{tabular}

$\uparrow:$ signifikant höher in der exponierten Gruppe; $\downarrow$ : signifikant erniedrigt in der exponierten Gruppe; WMD: gewichtete mittlere Differenz; SMD: standardisierte mittlere Differenz; $I^{2}$ = Inkonsistenzmaß, REM = Random-Effekt-Modell, FEM = fixiertes Effektmodell. 


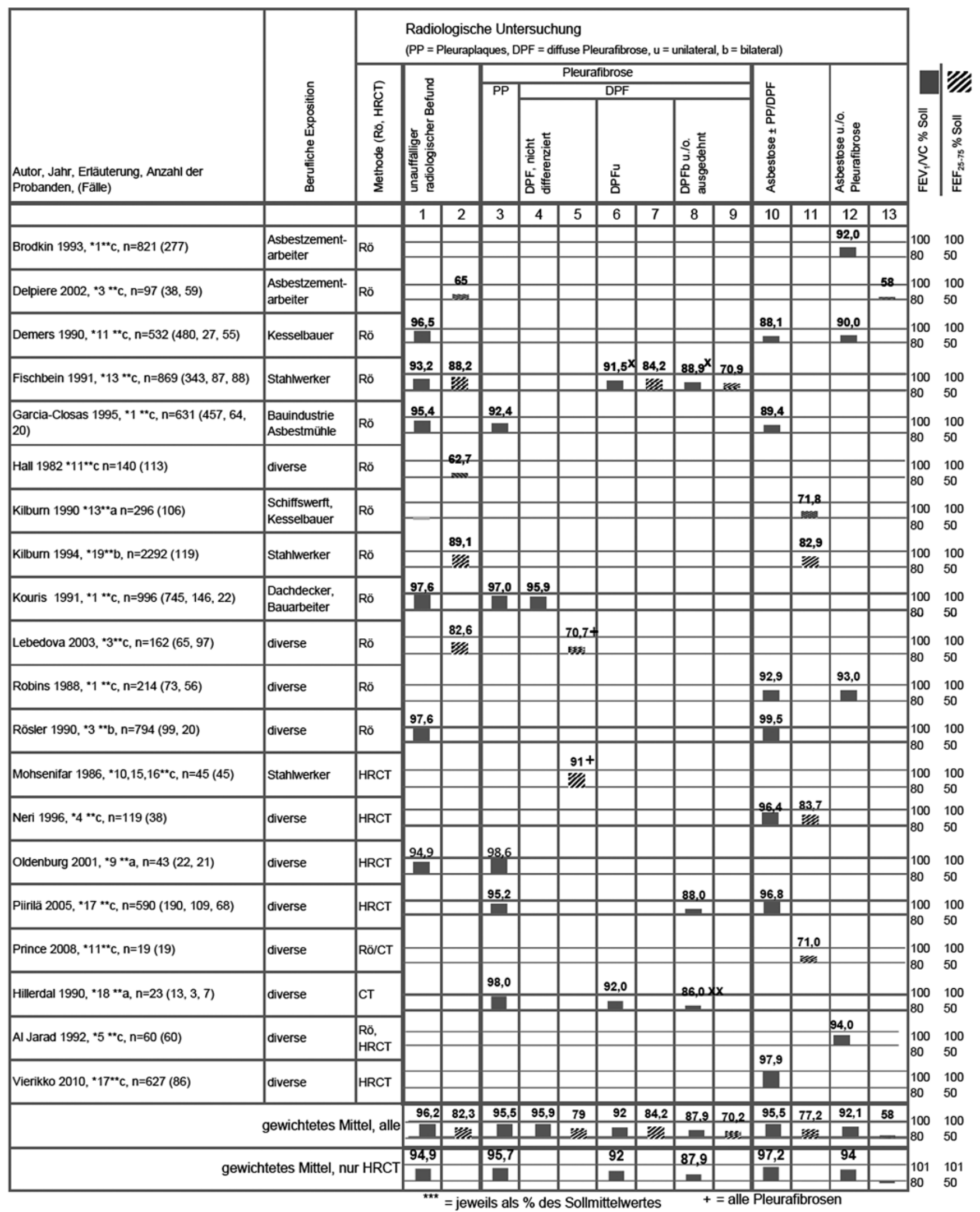

Abb. 1 FEV 1 /VG- und FEF $25-75$-Mittelwerte*** in verschiedenen Studien von asbestexponierten Probanden in Abhängigkeit vom radiologischen Befund.

\begin{tabular}{|c|c|c|c|}
\hline " ${ }^{*}$ Lungenfunktionsbezugswerte: & 04. Paoletti 1985 & 17. Viljanen 1982 & c) nicht differenziert, aber mit \\
\hline 01. Crapo 1981 & 05. Cotes 1979 & 18. Hedenström 1986 & Informationen über den Raucher- \\
\hline 03. Quanjer 1993 [Cave: Diese FEV ${ }^{-}$ & 09. Brändli 1996 & 19. Miller 1986 & status \\
\hline und FVC-Bezugswerte sind $>10 \% \mathrm{zu}$ & 10. Goldman und Becklake 1959 & & $\mathrm{x}=$ Inkludiert auch PP ohne DPF \\
\hline niedrig (Roberts, MacRae et al. 1991; & 11. Knudson 1983 & ${ }^{* *}$ Berücksichtigung des Raucher- & (geht nicht in die Berechnung des \\
\hline Baur, Isringhausen-Bley et al. 1999; & 13. Miller 1980 & status: & gewichteten Mittels ein) \\
\hline Hankinson, Odencrantz et al. 1999; & 15. Boren 1966 & a) Adjustierung des Raucherstatus & $\mathrm{xx}=$ Inkludiert je $1 \mathrm{x}$ ILO $1 / 0$ und \\
\hline Koch, Schaper et al. 2009)] & 16. Schmidt 1973 & b) nur Nieraucher & ILO $1 / 1$ \\
\hline
\end{tabular}




\section{a) Asbestexponierte ohne radiologisch fassbare Pleura- und Lungenveränderungen}

Eine Reihe von Autoren beschrieben signifikante oder zumindest trendmäßige Verminderungen von $\mathrm{FEF}_{25-75}$, so Delpierre et al. [60] (signifikant), Wang et al. [61] (signifikant, dabei auch signifikanter jährlicher Abfall), Fischbein et al. [54] (Reduktion auf 88,2 $\pm 32,5 \%$ ), Jodoin et al. [62] (n. s.; Abnahme der FEF-Werte um 11 20\%), Kennedy et al.[24], Kilburn und Warshaw [13] (Nichtraucherkollektiv 89,9\%, Exraucher $78,2 \%$ und aktuelle Raucher $71,8 \%$, in letzteren beiden Kollektiven signifikant erniedrigt; auBerdem signifikante $\mathrm{FEF}_{75-85}$-Verminderungen), Begin et al. [63] (n. s.), Lebedova et al. [38] $(82,6 \pm 27,1 \%$, pathologische Werte in $32,2 \%$ sowie von $\mathrm{FEF}_{75} 69,8 \pm 29,6 \%$, pathologische Werte in $53,8 \%$ ), Wang et al. [61] (signifikant), Neri et al. [40] (92,5 $\pm 30,2 \%$ ), Begin et al. [64] (nicht signifikant).

Im Kollektiv ohne radiologisch fassbare Veränderungen identifizierten Van Cleemput et al. [47] eine auf 80,9\% reduzierte $\mathrm{FEF}_{25}$. Oldenburg et al. [65] fanden eine Verminderung von $\mathrm{FEF}_{75}$, diskret auch von $\mathrm{FEF}_{50}$, ähnlich Rösler und Woitowitz [66] in Bezug auf die $\mathrm{FEF}_{50}$. Von Rosenstock et al. [21] wurde in 21,7\% eine Obstruktion (isoliert oder kombiniert) mitgeteilt.

Die nach langjähriger Asbestexposition feststellbaren Veränderungen im Sinne einer restriktiven Ventilationseinschränkung besitzen typischerweise auch eine obstruktive Komponente, welche durch erhöhte Isoflow-Werte (Zunahme der Atemflüsse unter Atmung mit einem Helium-Sauerstoffgemisch, d.h. geringerer Dichte) und einem erhöhten endexspiratorischen Atemwegswiderstand gekennzeichnet sind [67]. Begin et al. [64] beschreiben einen verminderten Isoflow unter Asbest-Arbeitern (n. s.). Kennedy et al. [24] beobachteten im Gesamtkollektiv und bei Obliteration des kostophrenischen Winkels signifikante $\mathrm{FEV}_{1} / \mathrm{FVC}$ und $\mathrm{FEF}_{25-75}$-Erniedrigungen; die Unterschiede waren nicht signifikant bei fehlender Beteiligung des kostophrenischen Winkels.

\section{b) Asbestexponierte mit Pleurafibrose}

Lebedova et al. [38] fanden signifikante $\mathrm{FEF}_{25-75}$-Verminderungen (50,5\% der Probanden der letzteren Autoren hatten pathologische Werte, wobei auch die $\mathrm{FEF}_{75}$ signifikant erniedrigt war).

In der Studie von Mohsenifar et al. [16] betrug die $\mathrm{FEF}_{25-75} 91$ $\pm 29 \%$ des Sollmittelwertes. Entsprechende Befunde erhoben Van Cleemput et al. [47] ( $\left.\mathrm{FEF}_{75} 84,8 \%\right)$, Hedenstierna et al. [68] (signifikant erniedrigte $\mathrm{FEF}_{50}$ und $\mathrm{FEF}_{75}$ ), Hillerdal et al. [69] $\left(\mathrm{FEF}_{50}\right.$ im Teilkollektiv mit bilateraler diffuser Pleurafibrose auf $50 \%$ signifikant vermindert; $\mathrm{FEF}_{50}$ bei unilateraler diffuser Pleurafibrose und Pleuraplaques jeweils $85 \%$ ).

\section{c) Asbestexponierte mit Asbestose}

Angaben zu FEF $25-75$ finden sich bei Kennedy et al. [24], Prince et al. [70] (in beiden Arbeiten signifikant vermindert), Kilburn und Warshaw [13] (82,9\% bei Nichtrauchern, 71\% bei Exrauchern und 70\% bei aktuellen Rauchern, letztere jeweils signifikant; ebenfalls war $\mathrm{FEF}_{75-85}$ sowohl bei Nichtrauchern, Exrauchern und aktuellen Rauchern signifikant erniedrigt), Begin et al. [63] (n. s.), Begin et al. [64] (ca. 70\%; n. s.) sowie Neri et al. [40] (83,7 $\pm 33,4 \%$; n. s.). Einschränkungen der $\mathrm{FEF}_{50}$ werden von Paris et al. [71], (80,7 $\pm 30 \%)$ sowie Rösler und Woitowitz [66], $(70,6 \pm 5,9 \%$; signifikant) beschrieben.

\section{d) Asbestexponierte mit Asbestose und/oder}

Pleuraveränderungen

In den Untersuchungen von Delpierre et al. [60] war $\mathrm{FEF}_{25-75}$ auf $65 \pm 58 \%$ signifikant erniedrigt und die Resistance auf 0,292 $\pm 0,023 \mathrm{kPa} / \mathrm{l} / \mathrm{s}$ signifikant erhöht, wobei in $43 \%$ eine Reversibilität der Obstruktion bestand.

Niebecker et al. [14] zeigten, dass die Resistance bei höherwertigen Asbestose- und Pleurabefunden signifikant erhöht war, wobei auch signifikante Unterschiede zwischen Nichtrauchern und Rauchern (ähnlich wie bei der $\mathrm{FEV}_{1} / \mathrm{FVC}$ ) bestanden.

\section{Synergistische Wirkung der Asbestbelastung und des Rauchens}

Die bereits erwähnte Metaanalyse von Filipelli et al. [19] weist auf signifikante synergistische Effekte des Rauchens in asbestexponierten Kollektiven hin (sowohl bzgl. $\mathrm{FEF}_{25-75}$ als auch TGV, RV/TGV und Resistance), wobei die Effekte des Rauchens auf die exspiratorischen Flüsse und die der Asbestexposition auf die Parameter der großen Atemwege dominieren (vgl. 0 Tab.1 b und $\odot$ Tab. 1c).

Entsprechende Ergebnisse werden in weiteren Arbeiten mitgeteilt, so von McDonald et al. [20], Dement et al. [6], Meldrum et al. [72], Chien et al. [22], Glencross et al. [23], Alfonso et al. [73], Begin et al. [74] (letztere mit einer Dosis-Wirkungsbeziehung hinsichtlich der $\mathrm{FEV}_{1}$-Abnahme).

Auch Bagatin et al. [30] konnten eine signifikante Assoziation zwischen obstruktiver Ventilationsstörung $\left(\mathrm{FEV}_{1} / \mathrm{FVC}<70 \%\right)$ und Rauchen unter Asbestarbeitern nachweisen (OR 1,56; $95 \%$ CI 1,30-1,86); diese war bemerkenswerterweise aber schwächer ausgeprägt als die Assoziation der Obstruktion mit Asbestose und asbestbedingten Pleuraveränderungen.

Ähnlich beschrieben Niebecker et al. [14] bei Vorliegen von mittel- und v. a. höhergradigen Pleura- und/oder Lungenbefunden unter Rauchern eine signifikant verminderte $\mathrm{FEV}_{1} / \mathrm{FVC}$ und eine signifikant erhöhte Resistance.

Sue et al. [15] zeigten, dass sowohl die $\mathrm{FEV}_{1} / \mathrm{FVC}$ - als auch $\mathrm{FEF}_{25-75^{-}}$ Einschränkungen unter rauchenden Asbestarbeitern (86,5\% bzw. $69,7 \%)$ signifikant stärker ausgeprägt waren als unter nichtrauchenden (95,3\% bzw. 97\%).

Neri et al. [40] wiesen im Gesamtkollektiv und auch im Teilkollektiv der Nichtraucher leichte Verminderungen von $\mathrm{FEF}_{25-75}$ (im Mittel von 11 bzw. 8\%) nach. Lebedova et al. [38] stellten eine signifikante $\mathrm{FEF}_{75^{-}}$und eine nicht ganz signifikante $\mathrm{FEF}_{25-75^{-}}$ Abnahme unter rauchenden Asbestarbeitern fest.

Oldenburg et al. [65] fanden in dem Kollektiv mit Pleuraveränderungen nur unter Rauchern und Exrauchern Einschränkungen der $F E V_{1} / F V C$ und $\mathrm{FEF}_{50}$ (beide n.s.). Nieraucher hatten überdurchschnittlich gute Werte; dieses spricht für einen Healthyworker-Effekt und damit eine Unterschätzung des Risikos.

Ähnliche Befunde erhoben Kilburn et al. [75] $\left(\mathrm{FEV}_{1} / \mathrm{VC} 75,6 \%\right.$, 70,2\%, 67,8\%; $\mathrm{FEF}_{25-75} 89,1 \%, 78,2 \%$ 71,8\%; $\mathrm{FEF}_{75-85} 95,4 \%$, 67,5\%, 81,5\%; jeweils Nichtraucher, Exraucher, aktive Raucher in Bezug auf die Sollmittelwerte).

Auch Kilburn und Warshaw [13] beschrieben nur unter früher rauchenden Asbestarbeitern mit Pleuraveränderungen oder Asbestose $\mathrm{FEV}_{1} / \mathrm{FVC}$-Einschränkungen; $\mathrm{FEF}_{75-85}$ war im Vergleich zu Nierauchern fünfmal stärker unter Exrauchern und viermal stärker unter aktuellen Rauchern reduziert.

Gleichartige Befunde erhoben Ohlson et al. [41]: Die FVC-Abnahmen betrugen $8,53 \%$ bzw. 3,79\%, die $\mathrm{FEV}_{1}$-Abnahmen 9,41\% bzw. $3,66 \%$ (jeweils Raucher bzw. Nichtraucher). 
Diese vorgenannten Aussagen werden auch von Ohar et al. [8] bestätigt: Die $\mathrm{FEV}_{1} / \mathrm{FVC}$ betrug $76,8 \pm 8,9 \%$ unter Nichtrauchern, $71,0 \pm 12,3 \%$ unter Exrauchern und $68,7 \pm 12,7 \%$ unter aktiven Rauchern $(\mathrm{p}<0,001)$.

\section{Dosis-Wirkungsbeziehungen und Latenzzeit}

Bagatin et al. [30] fanden in einer der umfangreichsten Studien an 3634 brasilianischen Chrysotil-Minenarbeitern, die zwischen 1940 und 1996 beschäftigt waren, eine signifikante Zunahme obstruktiver Ventilationsstörungen bei Vorliegen parenchymatöser oder pleuraler Veränderungen (neben einer Zunahme restriktiver Ventilationsstörungen). Dabei waren $\mathrm{FEV}_{1} / \mathrm{FVC}$ und $\mathrm{FEF}_{25-75}$ umso mehr eingeschränkt, je höher die Asbestdosis der einzelnen Gruppe war; allerdings ergab die multiple Regressionsanalyse keine signifikante Korrelation mit der kumulativen Asbestfaserbelastung.

\section{Dosis-Wirkungsbeziehungen}

Mehrere weitere Autoren fanden signifikante Dosis-Wirkungsbeziehungen [23,31].

Chien et al. [22] eruierten in einem 20-jährigen Follow-up eine signifikante Assoziation zwischen initialer $\mathrm{FEV}_{1} / \mathrm{FVC}$-Einschränkung und einer raschen weiteren Verschlechterung.

Moshammer und Neuberger [59] ermittelten eine signifikante Korrelation zwischen Asbestfaserdosis und $\mathrm{FEF}_{75}$-Abnahme.

Über eine Abhängigkeit von der Expositionsdauer (Dosis) berichten auch Ohlson et al. 1985 [41] (stärkerer Abfall der FEV Fls $_{1}$ aler FVC in den Teilkollektiven mit einer Belastung von $>15$ Faserjahren). Hunting und Welch [76] beschreiben eine Zunahme obstruktiver Atemwegserkrankungen nach mindestens 10jähriger Expositionszeit (OR 1,36, 95\% CI 0,43-4,28).

\section{Diskussion}

Mehrere bevölkerungsbezogene Studien weisen auf Überhäufigkeiten der COPD unter asbestexponierten Kollektiven hin, wobei allerdings meist keine detaillierten Angaben über die stattgefundene Belastung vorliegen [5,6,77-78].

Diese Literaturaus- und -bewertung belegt, dass die langjährige Asbestexposition mit einer offensichtlich von der AsbestfaserDosis, wahrscheinlich auch von der Latenzzeit abhängigen, überwiegend leichten obstruktiven Ventilationseinschränkung einhergeht. Entsprechende Befunde werden bereits bei normalem Röntgenthoraxbefund beobachtet (vgl. Kapitel 3 a, ferner Ohar et al. [8], Chien et al. [22], Demers et al. [31], Garcia-Closas et al. [55], Kouris et al. [79].

In der Mehrzahl der Veröffentlichungen wird eine leichte Zunahme der Obstruktionsparameter bei Vorliegen pleuraler Fibrosierungen mitgeteilt (vgl. Kapitel 2 und 3), teilweise mit positiven Assoziationen zwischen den Ausmaßen der radiologisch und funktionell erfassbaren Veränderungen (s. z.B. Fischbein et al. [54], Hillerdal et al. [69], Kouris et al. [79], Ohlson et al. [41], Piirilä et al. [80], Schwartz et al. [25], Shih et al. [53], Cotes und King [81], Robins und Green [82].

In den Kollektiven mit Asbestose zeigen sich im Mittel ähnliche Befunde wie im Kollektiv mit pleuralen Veränderungen, allerdings sind die Daten heterogener (vgl. Kapitel 2 und 3).

Ohar et al. [8] beschrieben ohne Bezug zum radiologischen Befund in einer 3383 ehemalige Asbestarbeiter umfassenden Kohorte mit einer Latenzzeit von $41 \pm 10$ Jahren in $25,4 \%$ eine obstruktive und in $6 \%$ eine kombinierte Ventilationsstörung. Diese
Arbeit, die allerdings einige Inkonsistenzen aufweist [12], betont ebenso wie jene von Harber et al. [83] die Unterschätzung der Obstruktion in Folge der Lungenblähung.

Delpierre et al. [60] werteten in einer retrospektiven Studie Bronchodilatationsdaten aus. Dabei ergab sich in 43\% der Asbestexponierten, die zu 65\% eine reduzierte $\mathrm{FEV}_{1}$ und $\mathrm{FEV}_{1} / \mathrm{FVC}$ aufwiesen (unabhängig davon, ob röntgenologisch fassbare Veränderungen vorlagen), ein mehr als $10 \%$ iger $\mathrm{FEV}_{1}$-Anstieg oder ein mindestens $50 \%$ iger $\mathrm{R}_{\mathrm{aw}}$-Abfall.

Hinzuweisen ist auf z. T. normale Obstruktionsparameter bei Vorliegen einer Asbestose [27,46,48,52]. Entsprechendes gilt für einige Veröffentlichungen, die sich auf Kollektive mit Pleurafibrosen beziehen [47,50-51]. Die meisten der vorgenannten Autoren verwendeten allerdings die nicht zeitgemäßen Lungenfunktionssollwerte von Quanjer [84] (basieren auf Studien der 1960er- und 1970er-Jahre mit mindestens $10 \%$ zu niedrigen

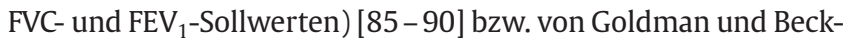
lake [91]; Entsprechendes trifft auch zu für Schwartz et al. $[25,48,52]$ und Shih et al. [53].

Jones et al. [11] beschreiben in dem von Kilburn und Warshaw [13] untersuchten Kollektiv unter Bezug auf die Referenzwerte von Morris et al. [92] deutlich geringere Abweichungen der Lungenfunktionsdaten als die Orginalarbeit. Smith [12] weist in einem Leserbrief hinsichtlich der Arbeit von Ohar et al. [8] auf Fehlermöglichkeiten der zu Grunde gelegten statistischen Verfahren hin.

Daneben können Healthy-worker-Effekte und unterschiedliche Grade der peribronchialen und interstitiellen Fibrosierung in den untersuchten Kollektiven sowie Confounder (andere intensive berufliche Staubbelastungen, unterschiedliches Rauchverhalten) für abweichende Befunde verantwortlich sein.

Nicht auszuschließen sind ferner Diskrepanzen zwischen den einzelnen Studien infolge unterschiedlicher Qualifikation des untersuchenden Personals sowie methodisch und gerätetechnisch bedingte Abweichungen.

Schließlich ergeben sich in mehreren Arbeiten Hinweise auf einen biphasischen Verlauf der Obstruktionsparameter mit einem frühen Beginn, aber anschließend bzw. dann bei höhergradigen radiologischen Befunden beobachtbarer rückläufiger Obstruktion. So fiel in den Untersuchungen von Dujic et al. [93] im Verlauf von 10 Jahren $\mathrm{FEF}_{25-75}$ von $100 \%$ auf $90 \%$ des Sollmittelwertes ab und stieg dann wieder in den folgenden 10 Jahren auf $100 \%$ an. Dies kann, ebenso wie ein ähnlicher Befund weiterer Autoren [28,41,50-51,94], durch eine fibrosebedingte Stabilisierung der Atemwege bedingt sein.

Weder in den hier dargestellten Studien noch in einer früheren Übersichtsarbeit [17] unterschied sich die Funktionseinschränkung grundsätzlich zwischen den Diagnosen auf konventionellradiologischer Basis und jenen, die mittels (HR)CT ermittelt wurden.

Auch fanden wir keinen Hinweis darauf, dass zwischen den verschiedenen Berufsgruppen mit Asbestexposition wesentliche Abweichungen in den Funktionseinschränkungen vorliegen.

Das Ergebnis unserer Literaturaus- und -bewertung wird in der Metaanalyse von Filippelli et al. [19] bestätigt; diese berücksichtigt allerdings nicht den radiologischen Befund und die Expositionsangaben. Die asbestexponierten Kollektive zeigen signifikante Abweichungen von $\mathrm{FEV}_{1} / \mathrm{FVC}(\mathrm{p}=0,003), \mathrm{FEF}_{25-75}$ $(p=0,002), R V / T G V(p<0,001)$ und Resistance $(p=0,001)$, wobei die mittlere Differenz von $\mathrm{FEV}_{1} / \mathrm{FVC}$ und Resistance unter den Asbestexponierten relativ stark durch die Asbestexposition und nicht eindeutig durch das Rauchen abweicht, andererseits die 
mittlere Differenz von $\mathrm{FEF}_{25-75}$ und v. a. von $\mathrm{FEV}_{75}[38,74]$ stärker durch das Rauchen als die Asbestexposition vermindert wurde. Im Mittel bewegen sich die Sollwertabweichungen der einzelnen Lungenfunktionsparameter im unteren Norm- bzw. Grenzbereich. Im Einzelfall sind die stattgefundene Belastungsdosis, die synergistischen Effekte des Rauchens und die individuelle Suszeptibilität (vgl. die Streuung in den Kollektiven) zu berücksichtigen. Es bestätigt sich, dass jeder Einzelfall einer eingehenden Prüfung inklusive einer umfassenden Lungenfunktionsdiagnostik bedarf, deren Ergebnis im Verlauf (vgl. Voruntersuchungen) beurteilt werden sollte [95].

Der adverse Effekt der Asbestexposition auf die Lungenfunktion ist pathophysiologisch nicht ausschließlich als Folge einer unspezifischen allgemeinen staubinduzierten Atemwegsinflammation darzustellen. Hinzu kommen komplexe interstitielle Prozesse, die mit multiplen Zytokinfreisetzungen, Bildung von Sauerstoffradikalen und peribronchialer und interstitieller Fibrose, konsekutiven, bereits früh einsetzenden Beeinträchtigungen der Diffusion und Atemmechanik im Sinne einer kombinierten Ventilationsstörung mit Gasaustauschstörung einhergehen.

Rauchen weist nach den übereinstimmenden Daten der Metaanalyse von Filippelli et al. [19] und Ergebnissen weiterer Autoren synergistische Effekte zum Asbest auf und steht hierbei teilweise im Vordergrund $[8,14,23,74]$. Die in den meisten Untersuchungen ausgeprägtere Einschränkung der mittel- und endexpiratori-

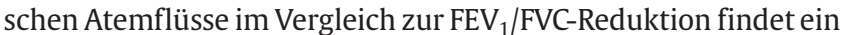
Korrelat in den pathologisch-anatomisch objektivierten Veränderungen i. S. eines Small airways diseases [96]. Bemerkenswerterweise geht Rauchen nach Untersuchungen von Churg and Stevens [97] mit einer 6-50fach höheren Asbestfaserdeposition im Bereich der Bronchioli respiratorii und der Alveolen einher.

Zusammenfassend ergibt diese auch in unserer ergänzenden Metaanalyse [98] bestätigte Literaturauswertung, dass die langjährige intensive Exposition gegenüber asbesthaltigen Stäuben nicht nur eine restriktive, sondern auch eine obstruktive Ventilationseinschränkung bewirkt. Dabei ergeben sich bzgl. der Letzteren synergistische Einflüsse durch das Rauchen, Irritanzien und nicht-asbesthaltige Staubanteile, die praktisch immer zusätzlich einwirken und sich in ihrer Wirkung oft kaum abgrenzen lassen. Die Reduktion aller Lungenfunktionsparameter ist in der überwiegenden Mehrzahl der Studien bereits ohne pathologischen Pleura- und Lungenparenchymbefund im konventionellen Röntgenbild und im CT festzustellen; im Allgemeinen nimmt die Funktionseinschränkung mit zunehmendem radiologischen Befund zu. Bourbeau et al. [26] stellen treffend fest: „Isolated pleural plaques are associated with a significant reduction in $\mathrm{FEV}_{1}$ und FVC, which cannot be attributed to the presence of radiographic and subradiographic pulmonary fibrosis“.

\section{Interessenkonflikt}

Die Autoren geben an, dass kein Interessenkonflikt besteht.

\section{Literatur}

1 Blanc PD, Menezes AM, Plana E et al. Occupational exposures and COPD: an ecological analysis of international data. Eur Respir J 2009; 33: $298-304$

2 Groneberg DA, Morfeld P, Kraus T et al. Health effects of particulate matter exposure: current scientific knowledge. Pneumologie 2009; 63: $363-368$
3 Pope CA 3rd. Mortality effects of longer term exposures to fine particulate air pollution: review of recent epidemiological evidence. Inhal Toxicol 2007; 19 Suppl 1: 33-38

4 Laden F, Schwartz J, Speizer FE, Dockery DW. Reduction in fine particulate air pollution and mortality: Extended follow-up of the Harvard Six Cities study. Am J Respir Crit Care Med 2006; 173: 667-672

5 Balmes J. Becklake M, Blanc P et al. American Thoracic Society Statement: Occupational contribution to the burden of airway disease. Am J Respir Crit Care Med 2003; 167: 787 - 797

6 Dement JM, Welch L, Ringen $K$ et al. Airways obstruction among older construction and trade workers at Department of Energy nuclear sites. Am J Ind Med 2010; 53: 224-240

7 Antonescu-Turcu AL, Schapira RM. Parenchymal and airway diseases caused by asbestos. Current opinion in pulmonary medicine 2010; 16: $155-161$

8 Ohar J, Sterling DA, Bleecker E, Donohue J. Changing patterns in asbestos-induced lung disease. Chest 2004; 125: 744 - 753

9 Enright P. Comment on spirometry. J Occup Med 1987; 29: 842

10 Oliver LC, Eisen EA, Greene R, Sprince NL. Asbestos-related pleural plaques and lung function. Am J Ind Med 1988; 14: 649-656

11 Jones RN, Glindmeyer HW 3rd, Weill H. Review of the Kilburn and Warshaw Chest article-airways obstruction from asbestos exposure. Chest 1995; 107: 1727-1729

12 Smith $D D$. Failure to prove asbestos exposure produces obstructive lung disease. Chest 2004; 126: 1000

13 Kilburn KH, Warshaw RH. Airways obstruction from asbestos exposure. Effects of asbestosis and smoking. Chest 1994; 106: 1061 - 1070

14 Niebecker M, Smidt U, Gasthaus L, Worth G. [The incidence of airway obstruction in asbestosis]. Pneumologie 1995; 49: 20-26

15 Sue DY, Oren A, Hansen JE, Wasserman K. Lung function and exercise performance in smoking and nonsmoking asbestos-exposed workers. Am Rev Respir Dis 1985; 132: 612-618

16 Mohsenifar Z, Jasper AJ, Mahrer T, Koerner SK. Asbestos and airflow limitation. J Occup Med 1986; 28: 817-820

17 Baur X, Wilken D. Auswirkungen der Asbestfaserstaub-Exposition auf die Lungenfunktion - ein systematisches Review. Pneumologie 2010; 64: $81-110$

18 Wilken D. Lung function impairment in asbestos workers. Doctoral thesis. In. Hamburg: Universität Hamburg, 2010

19 Filippelli $C$, Martines $V$, Palitti $T$ et al. [Meta-analysis of respiratory function of workers exposed to asbestos]. G Ital Med Lav Ergon 2008; 30: $142-154$

20 McDonald JC, Becklake MR, Gibbs GW et al. The health of chrysotile asbestos mine and mill workers of Quebec. Arch Environ Health 1974; 28: $61-68$

21 Rosenstock L, Barnhart S, Heyer NJ et al. The relation among pulmonary function, chest roentgenographic abnormalities, and smoking status in an asbestos-exposed cohort. Am Rev Respir Dis 1988; 138: 272-277

22 Chien JW, Au DH, Barnett MJ, Goodman GE. Spirometry, rapid FEV1 decline, and lung cancer among asbestos exposed heavy smokers. COPD 2007; 4: 339-346

23 Glencross PM, Weinberg JM, Ibrahim JG, Christiani DC. Loss of lung function among sheet metal workers: ten-year study. Am J Ind Med 1997; 32: 460-466

24 Kennedy SM, Vedal S, Muller $N$ et al. Lung function and chest radiograph abnormalities among construction insulators. Am J Ind Med 1991; 20: $673-684$

25 Schwartz DA, Fuortes LJ, Galvin JR et al. Asbestos-induced pleural fibrosis and impaired lung function. Am Rev Respir Dis 1990; 141: 321 326

26 Bourbeau J, Ernst P, Chrome J et al. The relationship between respiratory impairment and asbestos-related pleural abnormality in an active work force. Am Rev Respir Dis 1990; 142: 837-842

27 Ameille J, Matrat M, Paris C et al. Asbestos-related pleural diseases: dimensional criteria are not appropriate to differentiate diffuse pleural thickening from pleural plaques. Am J Ind Med 2004; 45: 289-296

28 Tonori Y, Niitsuya M, Sato T et al. Relationship between chest X-ray findings and pulmonary function tests in dust workers. Ind Health 2005; 43: $256-266$

29 Begin R, Cantin A, Berthiaume Y et al. Clinical features to stage alveolitis in asbestos workers. Am J Ind Med 1985; 8: 521 -536

30 Bagatin E, Neder JA, Nery LE et al. Non-malignant consequences of decreasing asbestos exposure in the Brazil chrysotile mines and mills. Occup Environ Med 2005; 62: 381 - 389 
31 Demers RY, Neale AV, Robins T, Herman SC. Asbestos-related pulmonary disease in boilermakers. American Journal of Industrial Medicine 1990; 17: 327 - 339

32 Baker EL, Dagg T, Greene RE. Respiratory illness in the construction trades. I. The significance of asbestos-associated pleural disease among sheet metal workers. J Occup Med 1985; 27: 483-489

33 Begin R, Masse S, Dufresne A. Further information on aluminium inhalation in silicosis. Occup Environ Med 1995; 52: 778- 780

34 Brodkin CA, Barnhart S, Anderson G et al. Correlation between respiratory symptoms and pulmonary function in asbestos-exposed workers. Am Rev Respir Dis 1993; 148: 32 - 37

35 Copley SJ, Lee YC, Hansell DM et al. Asbestos-induced and smokingrelated disease: apportioning pulmonary function deficit by using thin-section CT. Radiology 2007; 242: 258-266

36 Harkin TJ, McGuinness $G$, Goldring $R$ et al. Differentiation of the ILO boundary chest roentgenograph $(0 / 1$ to $1 / 0)$ in asbestosis by high-resolution computed tomography scan, alveolitis, and respiratory impairment. J Occup Environ Med 1996; 38: 46- 52

37 Al Jarad NA, Wilkinson P, Pearson MC, Rudd RM. A new high resolution computed tomography scoring system for pulmonary fibrosis, pleural disease, and emphysema in patients with asbestos related disease. $\mathrm{Br} J$ Ind Med 1992; 49: $73-84$

38 Lebedova J, Dlouha B, Rychla L et al. Lung function impairment in relation to asbestos-induced pleural lesions with reference to the extent of the lesions and the initial parenchymal fibrosis. Scand J Work Environ Health 2003; 29: $388-395$

39 Nakadate T. Decline in annual lung function in workers exposed to asbestos with and without pre-existing fibrotic changes on chest radiography. Occup Environ Med 1995; 52: 368 - 373

40 Neri S, Boraschi P, Antonelli A et al. Pulmonary function, smoking habits, and high resolution computed tomography (HRCT) early abnormalities of lung and pleural fibrosis in shipyard workers exposed to asbestos. Am J Ind Med 1996; 30: 588 - 595

41 Ohlson CG, Bodin L, Rydman T, Hogstedt C. Ventilatory decrements in former asbestos cement workers: a four year follow up. Br J Ind Med 1985; 42: 612-616

42 Sette A, Neder JA, Nery LE et al. Thin-section CT abnormalities and pulmonary gas exchange impairment in workers exposed to asbestos. Radiology 2004; 232: 66 - 74

43 Vierikko T, Jarvenpaa $R$, Toivio $P$ et al. Clinical and HRCT screening of heavily asbestos-exposed workers. Int Arch Occup Environ Health 2010; 83: 47-54

44 Markowitz SB, Morabia A, Lilis $R$ et al. Clinical predictors of mortality from asbestosis in the North American Insulator Cohort, 1981 to 1991. Am J Respir Crit Care Med 1997; 156: 101-108

45 Zedda S, Aresini G, Ghezzi I, Sartorelli E. Lung function in relation to radiographic changes in asbestos workers. Respiration 1973; 30: 132 140

46 Begin R, Ostiguy G, Filion R et al. Computed tomography in the early detection of asbestosis. Br J Ind Med 1993; 50: 689-698

47 Van Cleemput J, De Raeve H, Verschakelen JA et al. Surface of localized pleural plaques quantitated by computed tomography scanning: no relation with cumulative asbestos exposure and no effect on lung function. Am J Respir Crit Care Med 2001; 163: 705 - 710

48 Schwartz DA, Davis CS, Merchant JA et al. Longitudinal changes in lung function among asbestos-exposed workers. Am J Respir Crit Care Med 1994; 150: 1243 - 1249

49 Ameille J, Letourneux M, Paris $C$ et al. Does Asbestos Exposure Cause Airway Obstruction, in the Absence of Confirmed Asbestosis? Am J Respir Crit Care Med 2010; [Epub ahead of print]:

50 Peric I, Arar D, Barisic I et al. Dynamics of the lung function in asbestos pleural disease. Arh Hig Rada Toksikol 2007; 58: 407 - 412

51 Petrovic $P$, Ostojic L, Peric I et al. Lung function changes in pleural asbestosis. Coll Antropol 2004; 28: 711 - 715

52 Schwartz DA, Galvin JR, Yagla SJ et al. Restrictive lung function and asbestos-induced pleural fibrosis. A quantitative approach. J Clin Invest 1993; 91: $2685-2692$

53 Shih JF, Wilson JS, Broderick A et al. Asbestos-induced pleural fibrosis and impaired exercise physiology. Chest 1994; 105: 1370-1376

54 Fischbein A, Luo JC, Rosenfeld S et al. Respiratory findings among ironworkers: results from a clinical survey in the New York metropolitan area and identification of health hazards from asbestos in place at work. Br J Ind Med 1991; 48: $404-411$

55 Garcia-Closas M, Christiani DC. Asbestos-related diseases in construction carpenters. Am J Ind Med 1995; 27: 15-125
56 Piirila P, Kivisaari L, Huuskonen 0 et al. Association of findings in flowvolume spirometry with high-resolution computed tomography signs in asbestos-exposed male workers. Clin Physiol Funct Imaging 2009; 29: $1-9$

57 Rui F, De Zotti R, Negro C, Bovenzi M. [A follow-up study of lung function among ex-asbestos workers with and without pleural plaques] Med Lav 2004; 95: 171 - 179

58 Miller A, Lilis R, Godbold J et al. Spirometric impairments in long-term insulators. Relationships to duration of exposure, smoking, and radiographic abnormalities. Chest 1994; 105: 175-182

59 Moshammer $H$, Neuberger $M$. Lung function predicts survival in a cohort of asbestos cement workers. Int Arch Occup Environ Health 2009; 82: $199-207$

60 Delpierre S, Delvolgo-Gori MJ, Faucher M, Jammes Y. High prevalence of reversible airway obstruction in asbestos-exposed workers. Arch Environ Health 2002; 57: $441-445$

61 Wang XR, Yano E, Wang $M$ et al. Pulmonary function in long-term asbestos workers in China. J Occup Environ Med 2001; 43: 623-629

62 Jodoin G, Gibbs GW, Macklem PT et al. Early effects of asbestos exposure on lung function. Am Rev Respir Dis 1971; 104: 525 - 535

63 Begin R, Filion R, Ostiguy G. Emphysema in silica- and asbestos-exposed workers seeking compensation. A CT scan study. Chest 1995; 108 647-655

64 Begin R, Cantin A, Berthiaume Yet al. Airway function in lifetime-nonsmoking older asbestos workers. Am J Med 1983; 75: 631 - 638

65 Oldenburg $M$, Degens $P$, Baur X. Asbest-bedingte Lungenfunktionseinschränkungen mit und ohne Pleuraplaques. Atemwegs- und Lungenkrankheiten 2001; 27: 422-423

66 Rösler JA, Woitowitz HJ. Lungenfunktionsveränderungen bei Nichtrauchern mit Asbeststaublungenerkrankungen. In: Schuckmann F, Schopper-Jochum J, Hrsg. Bericht über die 30. Jahrestagung der Deutschen Gesellschaft für Arbeitsmedizin. Stuttgart: Gentner, 1990: 113-118

67 Wright JL, Cagle P, Churg A et al. Diseases of the small airways. Am Rev Respir Dis 1992; 146: 240-262

68 Hedenstierna G, Alexandersson R, Kolmodin-Hedman B et al. Pleural plaques and lung function in construction workers exposed to asbestos. Eur J Respir Dis 1981; 62: 111-122

69 Hillerdal G, Malmberg P, Hemmingsson A. Asbestos-related lesions of the pleura: parietal plaques compared to diffuse thickening studied with chest roentgenography, computed tomography, lung function, and gas exchange. Am J Ind Med 1990; 18: 627 - 639

70 Prince $P$, Boulay ME, Page $N$ et al. Induced sputum markers of fibrosis and decline in pulmonary function in asbestosis and silicosis: a pilot study. Int J Tuberc Lung Dis 2008; 12: 813-819

71 Paris C, Benichou J, Raffaelli C et al. Factors associated with early-stage pulmonary fibrosis as determined by high-resolution computed tomography among persons occupationally exposed to asbestos. Scand J Work Environ Health 2004; 30: 206 - 214

72 Meldrum M, Rawbone R, Curran AD, Fishwick D. The role of occupation in the development of chronic obstructive pulmonary disease (COPD). Occup Environ Med 2005; 62: 212 - 214

73 Alfonso HS, Fritschi L, de Klerk NH et al. Effects of asbestos and smoking on the levels and rates of change of lung function in a crocidolite exposed cohort in Western Australia. Thorax 2004; 59: 1052 - 1056

74 Begin R, Boileau R, Peloquin S. Asbestos exposure, cigarette smoking, and airflow limitation in long-term Canadian chrysotile miners and millers. Am J Ind Med 1987; 11: 55 - 66

75 Kilburn KH, Warshaw RH, Einstein K, Bernstein J. Airway disease in nonsmoking asbestos workers. Arch Environ Health 1985; 40: $293-295$

76 Hunting KL, Welch $L S$. Occupational exposure to dust and lung disease among sheet metal workers. Br J Ind Med 1993; 50: 432 - 442

77 Lebowitz MD. Occupational exposures in relation to symptomatology and lung function in a community population. Environ Res $1977 ; 14$ : $59-67$

78 Viegi G, Prediletto R, Paoletti P et al. Respiratory effects of occupational exposure in a general population sample in north Italy. Am Rev Respir Dis 1991; 143: $510-515$

79 Kouris SP, Parker DL, Bender AP, Williams AN. Effects of asbestos-related pleural disease on pulmonary function. Scand J Work Environ Health 1991; 17: 179-183

80 Piirila P, Lindqvist M, Huuskonen O et al. Impairment of lung function in asbestos-exposed workers in relation to high-resolution computed tomography. Scand J Work Environ Health 2005; 31: 44-51 
81 Cotes JE, King B. Relationship of lung function to radiographic reading (ILO) in patients with asbestos related lung disease. Thorax 1988; 43: $777-783$

82 Robins TG, Green MA. Respiratory morbidity in workers exposed to asbestos in the primary manufacture of building materials. Am J Ind Med 1988; $14: 433-448$

83 Harber P, Tashkin DP, Lew M, Simmons M. Physiologic categorization of asbestos-exposed workers. Chest 1987; 92: 494-499

84 Quanjer PH. Standardized lung function testing. Bull Eur Physiopathol Respir 1983; 19: 1 - 96

85 Baur X, Isringhausen-Bley S, Degens P. Comparison of lung-function reference values. Int Arch Occup Environ Health 1999; 72: 69-83

86 Hankinson JL, Odencrantz JR, Fedan KB. Spirometric reference values from a sample of the general U.S. population. Am J Respir Crit Care Med 1999; 159: 179-187

87 Koch B, Schaper C, Ittermann T et al. [Reference values for lung function testing in adults-results from the study of health in Pomerania“ (SHIP)]. Dtsch Med Wochenschr 2009; 134: 2327-2332

88 Roberts CM, MacRae KD, Winning AJ et al. Reference values and prediction equations for normal lung function in a non-smoking white urban population. Thorax 1991; 46: 643-650

89 Karrasch S, Ernst K, Behr J et al. Lassen sich spirometrische Referenzwerte in das fortgeschrittene Alter extrapolieren? Pneumologie 2010; 64: S157-S260
90 Langhammer A, Johnsen R, Gulsvik A et al. Forced spirometry reference values for Norwegian adults: the Bronchial Obstruction in Nord-Trondelag Study. Eur Respir J 2001; 18: 770 - 779

91 Goldman HI, Becklake MR. Respiratory function tests; normal values at median altitudes and the prediction of normal results. Am Rev Tuberc 1959; 79: 457-467

92 Morris JF, Koski A, Johnson LC. Spirometric standards for healthy nonsmoking adults. Am Rev Respir Dis 1971; 103: 57-67

93 Dujic Z, Tocilj J, Boschi S et al. Biphasic lung diffusing capacity: detection of early asbestos induced changes in lung function. Br J Ind Med 1992; 49: $260-267$

94 Britton MG. Asbestos pleural disease. Br J Dis Chest 1982; 76: 1 - 10

95 Woitowitz HJ. Berufliche Asbeststaubexposition und obstruktive Ventilationsstörungen. Int Arch Arbeitsmed 1970; 27: 244-256

96 Churg A, Wright JL, Wiggs B et al. Small airway disease and mineral dust exposure. Prevalence structure and function. Am Rev Respir Dis 1985; 131: $139-143$

97 Churg A, Stevens B. Enhanced retention of asbestos fibers in the airways of human smokers. Am J Respir Crit Care Med 1995; 151: 1409-1413

98 Wilken D, Velasco Garrido M, Manuwald U, Baur X. Relationship between occupational exposure to asbestos, lung function and radiological findings. submitted 